\title{
ANALYSIS OF HIGH-SPEED CONTINUOUS CASTING WITH INVERSE FINITE ELEMENTS
}

\author{
N. MALAMATARIS AND T. C. PAPANASTASIOU* \\ Department of Chemical Engineering, The University of Michigan, Ann Arbor, MI 48109. U.S.A.
}

\begin{abstract}
SUMMARY
A recently proposed inverse isotherm finite element method is further extended in order to account for processes with distorted isotherms. With this method a variety of problems can be solved which require the explicit calculation of characteristic material lines along with the common field of unknowns in transport phenomena. The method is applied to high-speed metal casting, where the location and shape of the extensive solidification front is calculated simultaneously with the primary unknowns, the velocity and the pressure, whereas the temperature is fixed at the moving nodes of the finite element tessellation. This is achieved by solving the energy equation inversely along with the rest of the conservation equations, i.e. the temperature field is fixed and its location is calculated. Empirical correlations may be derived which give the shape of the solidification front as a function of the process parameters. This may be used to improve the control means of metal casting, which is currently based on one-dimensional approximate analyses.
\end{abstract}

KEY WORDS Inverse finite elements Metal casting Extrusion Solidification front

\section{INTRODUCTION}

In this work, high-speed non-isothermal extrusion of Newtonian liquids with solidification inside the die is analysed with the method of inverse finite elements, which is a good prototype of continuous steel casting. This is a highly non-linear problem owing to the dominant convective terms in the conservation equations and the presence of a free boundary, the solidification front, of unknown shape and location. In an extensive recent literature review by Alexandrou et al. ${ }^{1}$ an overview was given of the previous work in this area. A number of studies that analyse non-isothermal extrusion of Newtonian and non-Newtonian liquids do not deal with solidification. $^{2-5}$ Elsewhere, the problem of solidification is studied extensively independently of extrusion. ${ }^{6-9}$ The numerical methods used solve primarily linear Stefan-like problems where the solidification front is obtained by successive iterations of linear convergence. ${ }^{10-12}$ In a comprehensive paper by Ettourney and Brown, ${ }^{13}$ non-isothermal flow and solidification of Newtonian fluids was studied and iteration schemes were evaluated for cases where the non-linear convective terms in the equations of motion were neglected. Complete two-dimensional schemes for continuous casting were developed by Onoda ${ }^{14}$ and Alexandrou et al., ${ }^{1}$ where all non-linearities are addressed and all boundary conditions associated with phase change are satisfied directly at the solidification front. The developed algorithms were tested for Reynolds numbers up to 100

* Author to whom correspondance should be addressed.

0271-2091/91/201207-17\$08.50

(C) 1991 by John Wiley \& Sons, Ltd.

Received 11 February 1990

Revised 19 February 1991 
though, where the solidification front does not extend significantly far downstream from the die exit and its curvature is small. The latter case, although a good prototype for low-speed casting and polymer extrusion, is not representative for high-speed continuous casting at high Reynolds and Peclet numbers, where the solidification front may extend from 10 diameters inside the die exit up to 100 diameters downstream.

Commonly, the steel sheet is cut just downstream from the extreme end of the solidification front, where the material is still hot, being at a temperature just below its solidification temperature. In this way, flow of molten steel is avoided and energy to cut is saved. ${ }^{15}$ Therefore the control of the position and shape of the solidification front in terms of the external cooling is among the primary concerns in continuous casting. ${ }^{16}$ Developed control packages are based on one-dimensional heat conduction equations under the assumption of plug flow everywhere downstream from the attachment of the solidification front. ${ }^{16,17,18}$ Such one-dimensional analyses, though simple and efficient, may not be accurate enough. The present analysis provides the means for evaluation of such approximate one-dimensional analyses.

Recently, Alexandrou et al. ${ }^{1}$ proposed an inverse isotherm finite element method (IIFEM) to solve this problem. Additionally, the method has been shown to work in various other application problems with analytic solutions. ${ }^{19}$ The basic idea of the IIFEM is to discretize the flow domain into finite elements with perpendicular (across the flow) sides on isotherms. The energy equation which defines the position of the isotherms is then solved inversely simultaneously with the remaining conservation equations, which permits the simultaneous evaluation of the position of the isotherms along with the primary unknowns, the velocity and the pressure, while the temperatures are fixed along isotherms of unknown position. Some similarities exist between the IIFEM and the isotherm migration method, ${ }^{20}$ which is based on finite differences and the analytic inversion of a linear energy equation, used to solve moving boundary problems in one dimension. ${ }^{21}$

The IIFEM, as it stands, cannot be applied to certain processes of high Reynolds and Peclet numbers where the isotherms of the process are distorted to such a degree that problems can be caused in the convergence of the iteration. These problems are alleviated in this work by not requiring the transverse sides of all the finite elements to remain on isotherms while the inverse character is preserved. This is achieved by imposing a predefined temperature distribution on each of the transverse sides of the finite elements, rather than a unique temperature, while permitting their position to be updated by the Newton iteration of the inversely solved energy equation. Thus the isotherm character of the IIFEM is preserved only at the solidification front and its inverse character is preserved everywhere. This is an inverse finite element method (IFEM).

\section{GOVERNING EQUATIONS AND BOUNDARY CONDITIONS}

The new inverse method will be developed in connection with the process of metal casting, the geometry of which is shown in Figure 1. Molten metal is flowing into a vertical die (mould). It is being continuously cooled along the solid wall and the free surface (spray zone) by water. The solid metal slips perfectly along the wall, being drawn with a constant velocity (casting rate). The solidification front extends far into the solid region. The metal must be cut just downstream from the peak of the front, where the solid is soft, being still around its solidification temperature. The governing equations are made dimensionless by means of units of mould half-width $W$ for distance, of casting speed $V$ for velocity, of $\mu V / W$ units for pressure and of solidification temperature units $T_{\mathrm{s}}$ for temperature. The resulting equations in the liquid phase at steady state 


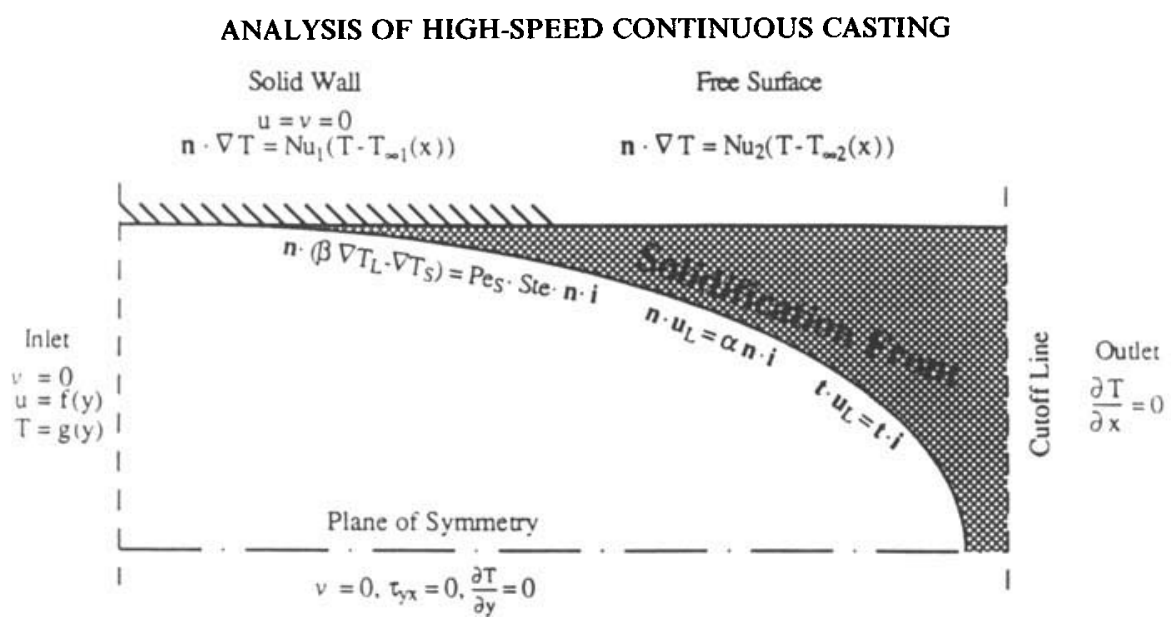

Figure 1. Metal-casting process: domain and boundary conditions

are

$$
\begin{gathered}
\nabla \cdot \mathbf{u}=0 \\
\operatorname{Re} \mathbf{u} \cdot \nabla \mathbf{u}=\nabla \cdot\left[-p \mathbf{I}+\nabla \mathbf{u}+(\nabla \mathbf{u})^{\mathrm{T}}\right]+S t \mathbf{k}, \\
P e_{\mathbf{L}} \mathbf{u} \cdot \nabla T=\nabla^{2} T+\operatorname{Br}\left[\nabla \mathbf{u}+(\nabla \mathbf{u})^{\mathrm{T}}\right]: \nabla \mathbf{u}
\end{gathered}
$$

and in the solid state

$$
P e_{\mathrm{S}} \mathbf{u} \cdot \nabla T=\nabla^{2} T
$$

Here $\mathbf{u}=(u, v)$ is the velocity vector of the fluid with $u$ and $v$ its components in the $x$ - and $y$-direction respectively, $p$ is the pressure, $T$ is the temperature, $I$ is the identity tensor and $\mathbf{k}$ is the unit vector in the direction of gravity (identical to the $x$-direction). $R e=\rho_{\mathrm{L}} V W / \mu$ is the Reynolds number, with $V$ the casting rate, $W$ the mould half-width, $\rho_{\mathrm{L}}$ the density and $\mu$ the viscosity of the molten metal. $S t=\rho_{\mathrm{L}} g L^{2} / \mu V$ is the Stokes number, with $g$ the acceleration of gravity. $P e_{\mathrm{L}}=\operatorname{Re} \operatorname{Pr}_{\mathrm{L}}$ is the Peclet number of the molten metal, where $\operatorname{Pr}_{\mathrm{L}}=c_{\mathrm{L}} \mu / k_{\mathrm{L}}$ is the Prandtl number of the molten metal, with $c_{\mathrm{L}}$ and $k_{\mathrm{L}}$ its heat capacity and heat conductivity respectively. $B r=\mu V^{2} / k_{\mathrm{L}} T_{\mathrm{s}}$ is the Brinkman number, with $T_{\mathrm{s}}$ the solidification temperature of the molten metal. $P e_{\mathrm{S}}=\operatorname{Re} P r_{\mathrm{S}}$ is the Peclet number of the solid metal, where $P r_{\mathrm{S}}=c_{\mathrm{S}} \mu \rho_{\mathrm{S}} / k_{\mathrm{S}} \rho_{\mathrm{L}}$, with $c_{\mathrm{S}}, \rho_{\mathrm{S}}$ and $k_{\mathrm{S}}$ the heat capacity, density and heat conductivity of the solid metal respectively. The properties of the molten metal are assumed to be constant. However, variations of them with respect to temperature can be easily taken into account by this model, as shown elsewhere. ${ }^{22}$ Predictions obtained with temperature-dependent density and viscosity differ only slightly from those with constant properties. This is consistent with the small sensitivity of the density and viscosity of steel to temperature within the real casting conditions considered in this work. ${ }^{23}$ The dimensionless boundary conditions of the process, shown in Figure 1, are

at the inlet

$$
\begin{aligned}
u(y) & =\frac{3}{2} \alpha\left(1-y^{2}\right), \\
v & =0, \\
T(y) & =C_{1} y^{n}+C_{2},
\end{aligned}
$$


along the solid wall

$$
\begin{aligned}
u & =0, \\
v & =0, \\
\left.(\partial T / \partial y)\right|_{y=1} & =-N u_{1}\left(T-T_{\infty 1}\right),
\end{aligned}
$$

along the free surface

$$
\left.(\partial T / \partial y)\right|_{y=1}=-N u_{2}\left(T-T_{\infty 2}\right),
$$

at the solidification front

$$
\begin{aligned}
\mathbf{n} \cdot \mathbf{u} & =\alpha \mathbf{n} \cdot \mathbf{i}, \\
\mathbf{t} \cdot \mathbf{u} & =\mathbf{t} \cdot \mathbf{i}, \\
\mathbf{n} \cdot\left(\beta \nabla T_{\mathrm{L}}-\nabla T_{\mathbf{S}}\right) & =P e_{\mathrm{S}} \text { Ste } \mathbf{n} \cdot \mathbf{i},
\end{aligned}
$$

at the midplane of symmetry

$$
\begin{array}{r}
\partial u / \partial y=0 \\
v=0 \\
\partial T / \partial y=0,
\end{array}
$$

at the synthetic outlet

$$
\partial T / \partial x=0 .
$$

At the inlet the flow of the molten metal is assumed to be laminar and fully developed and the temperature profile is known, equations (5)-(7). $\alpha=\rho_{\mathrm{S}} / \rho_{\mathrm{L}}$ is the ratio of the densities of the solid and the liquid metal, which appears in equation (12). At the solid wall there is no slip for the velocities, equations (8)-(9). The heat exchange along the wall and the free surface is given by Newton's law of cooling in equations (10) and (11) respectively. At the solidification front, equation (12) is due to conservation of mass, equation (13) is a kinematic condition of no slip in the tangential direction to the front and equation (14) is due to energy conservation. $\mathbf{n}$ and $\mathbf{t}$ are the unit normal and tangent to the solidification front and $\beta=k_{\mathrm{L}} / k_{\mathrm{S}}$ is the ratio of the heat conductivities. $N u_{1}=h_{1} W / k$ and $N u_{2}=h_{2} W / k$ are the Nusselt numbers of the mould and the free surface, with $h_{1}$ and $h_{2}$ the corresponding heat transfer coefficients, and Ste $=\Delta H_{\mathrm{f}} / c_{\mathrm{s}} T_{\mathrm{S}}$ is the Stefan number, where $\Delta H_{\mathrm{f}}$ is the latent heat of solidification of the metal. At the midplane, equations (15)-(17) are the appropriate symmetry conditions. At the synthetic outflow it is assumed that heat conduction in the $x$-direction is negligible.

\section{INVERSE FINITE ELEMENT FORMULATION}

The domain was tessellated into $27 \times 8$ rectangular finite elements as shown in Figure 2 . This tessellation gave macroscopic results independent of further refinement (maximum difference less than $10^{-3}$ ). As shown in Figure 3, the shape and position of the solidification front do not vary when a finer $27 \times 10$ rectangular mesh is used for the calculations. A typical run of the model on an IBM 3090-600E/MV computer takes approximately 10 CPU time seconds. Unfortunately, there are no similar approaches in the literature with which to compare. The unknown velocities $u$ and $v$ and the pressure $p$, which correspond to the continuity and momentum equations, 

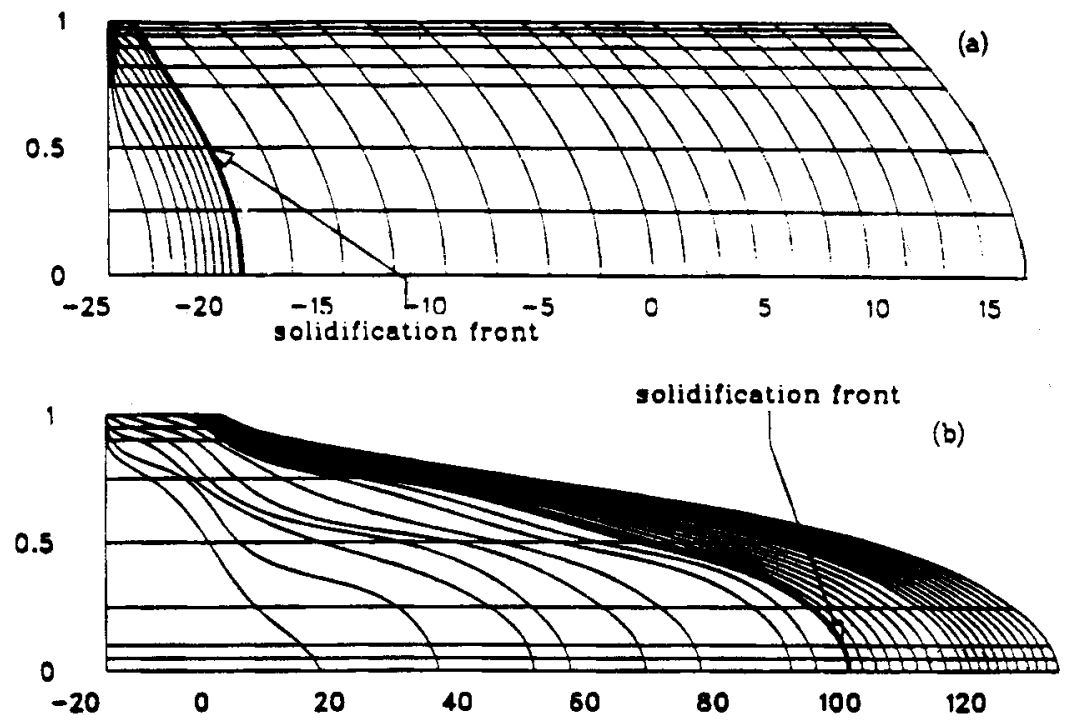

Figure 2. Typical finite element tessellations after Newton iteration convergence for (a) low-speed extrusion and (b) high-speed casting
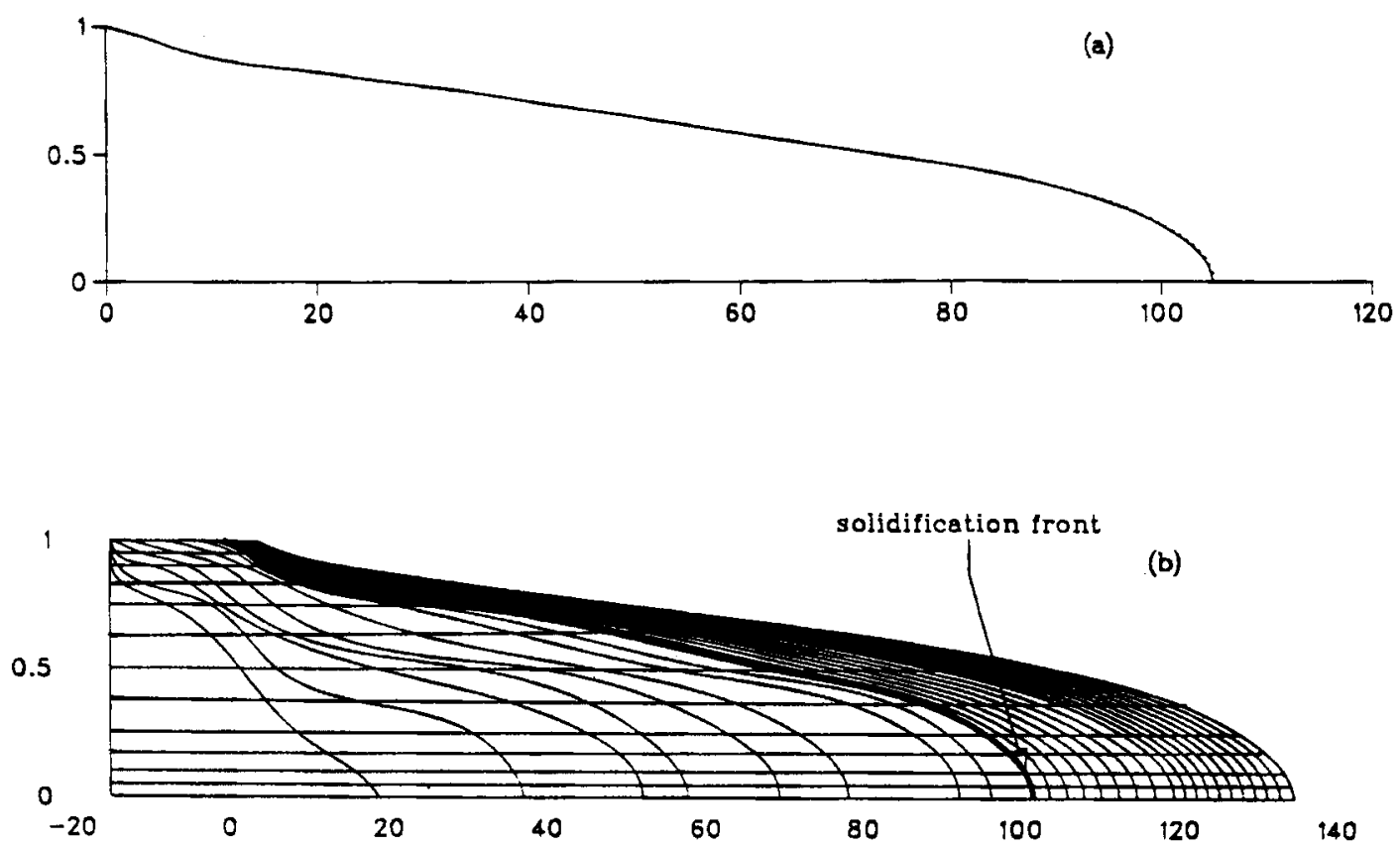

Figure 3. (a) Demonstration of mesh independence of the results of this work by comparing the solidification length obtained with a $27 \times 8(-)$ and a $27 \times 12(\cdots)$ mesh and (b) demonstration of the mesh tessellation of the finer mesh 
equations (1) and (2), are expanded in terms of Galerkin basis functions:

$$
u=\sum_{i=1}^{9} u_{i} \phi^{i}, \quad v=\sum_{i=1}^{9} v_{i} \phi^{i}, \quad p=\sum_{i=1}^{4} p_{i} \psi^{i},
$$

where $\phi^{i}$ are biquadratic and $\psi^{i}$ bilinear basis functions. These equations are solved with the ordinary finite element method where the $u_{i}, v_{i}$ and $p_{i}$ are calculated on the grid points of the discretized domain. In the inverse isotherm finite element method developed by Alexandrou $e t$ al. ${ }^{1}$ the energy equations, equations (3) and (4), are solved inversely, simultaneously with equations (1) and (2). In order to achieve this, the transverse sides of the elements are isotherms with preset values of temperature at each node and the unknowns are the $x$-positions of the nodes of the isotherms, expanded in terms of Galerkin quadratic basis functions as well:

$$
x=\sum_{i=1}^{9} x_{i} \phi^{i}
$$

The inverse solution of the energy equation, under the expansion by equation (20), allows the initially guessed isotherms of designated fixed temperature to update their position and shape as the Newton iteration progresses to a final configuration, which satisfies energy conservation, as shown in Figure 4. The inverse isotherm finite element method was found to perform satisfactorily in cases of relatively well-behaving isotherms. When the designated isotherms, which coincide

\section{- Initial Guess}

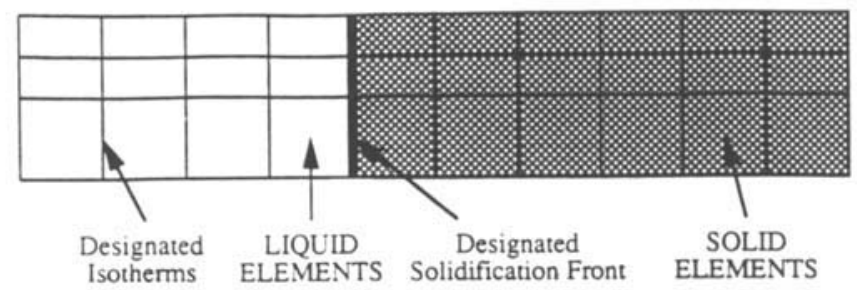

- First Newton Iteration

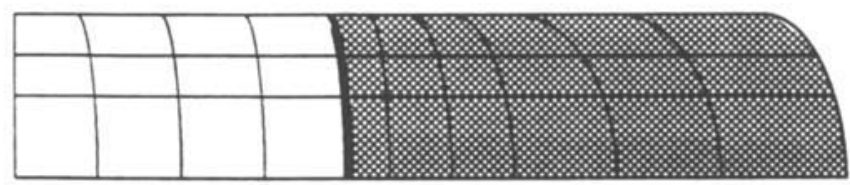

- Converged Solution

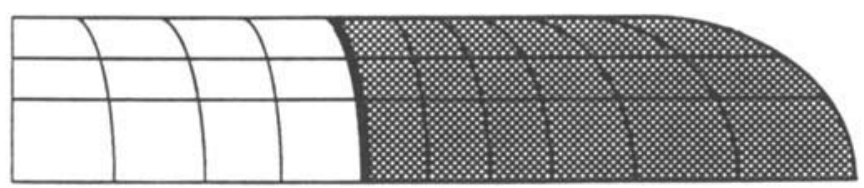

Figure 4. In the inverse isotherm finite element method (IIFEM) the position and shape of selected isotherms of known temperature are updated by Newton iteration to a final configuration satisfying the conservation equations 
with transverse element sides, are significantly distorted to configurations which are nearly parallel to the streamlines of the flow, the quadratic convergence of the Newton iteration deteriorates to no convergence at all. Although this is uncommon in slow extrusion of polymers, it is very likely to occur in metal casting of high speed and fast cooling, which results in an elongated solidification front and isotherms almost parallel to the casting direction, as shown in Figure 5. In fact, Figure 6 shows an actual run at high Reynolds numbers $(R e=1770)$ using the inverse isotherm finite element method, which incorrectly predicts isotherms intersecting each other owing to the extreme distortion of the finite elements, which eventually destroys the convergence of the Newton iteration.

In order to cure this pathology, the isotherm character of the inverse method is omitted for all isotherms but the solidification front. This relaxes the requirement that transverse sides of elements remain on isotherms and allows more flexibility in distributing elements to avoid large element aspect ratios. In the new inverse formulation this is achieved by associating nodes on transverse element sides with a predefined temperature, different in general from node to node. That is, adjacent nodes on the same transverse co-ordinate line are no longer required to be on the same isotherm and the energy equation is solved inversely for the unknown position of these nodes. As depicted in Figure 7, no a priori information, in addition to that needed for conventional finite element methods, about the temperature field is needed. The temperature at the inlet is known to be approximately $10 \%$ higher than the solidification temperature in casting operations. ${ }^{23}$ Since the molten metal is continuously cooled along the solid wall after it enters the mould, the temperature downstream from the inlet must have values less than the magnitude of the inlet temperature profile and greater than the solidus temperature. Nodal values on horizontal mesh lines are set by an interpolation scheme between the inlet and solidification temperatures and the inverse method calculates the location of the nodes where these temperatures occur. In this way any temperature field can in principle be accommodated, whereas with

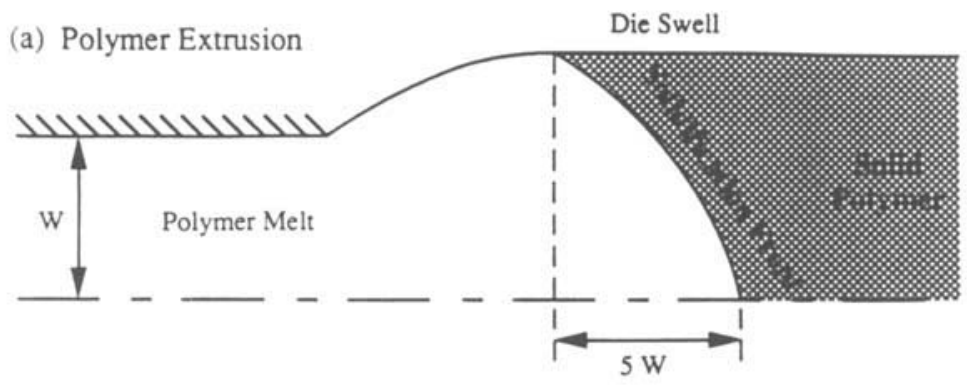

(b) Metal Casting

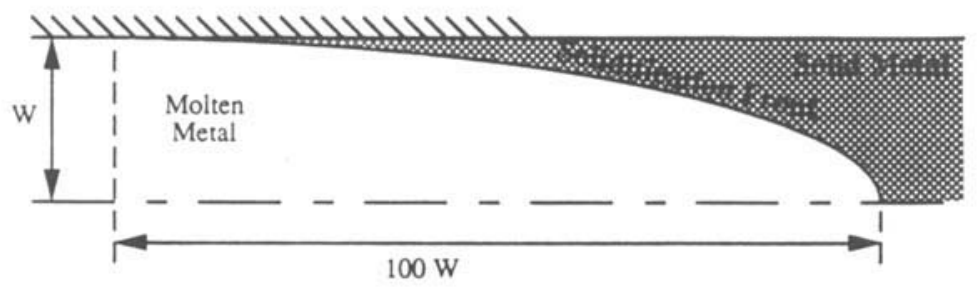

Figure 5. Illustration of the difference in curvature of solidification fronts depending on the nature of the process under investigation 


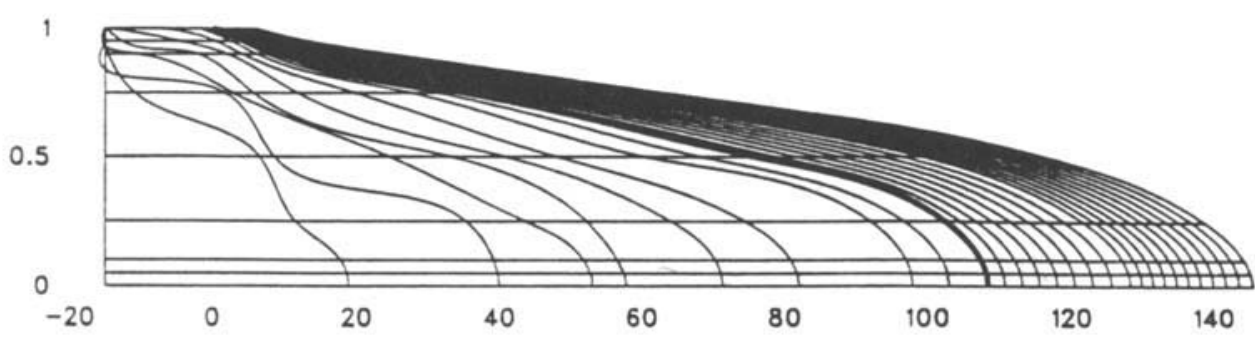

Figure 6. Demonstration of faulty intersection of isotherms by the inverse isotherm finite element method at $R e=1770$, $N u_{1}=2 \cdot 95$ and $N u_{2}=6 \cdot 1$

(a) Initial Guess

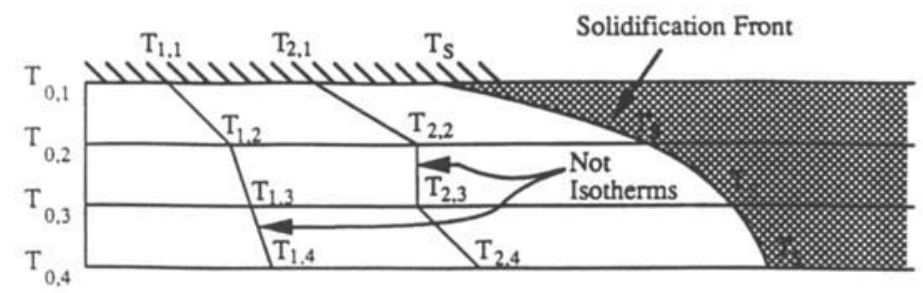

(b) After First Newton Iteration

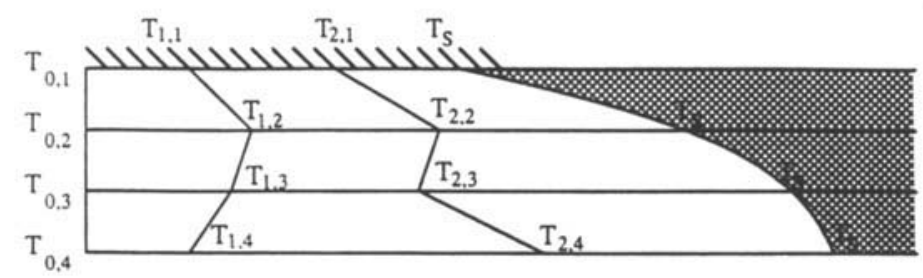

(c) After Convergence

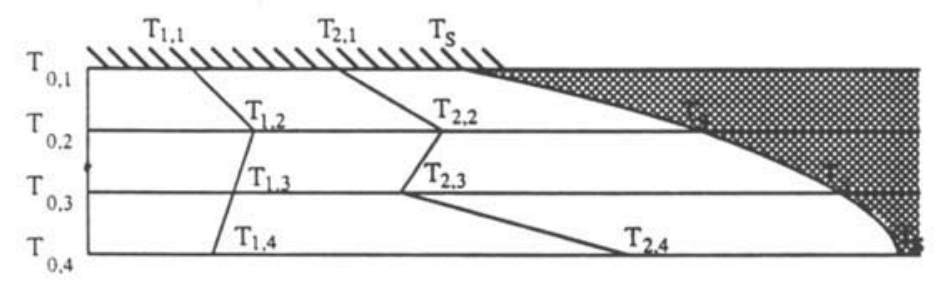

Figure 7. The modified inverse finite element method (IFEM), with the exception of the solidification front, does not require transverse sides of elements to remain on isotherms, which is the main difference from the inverse isotherm finite element method (IIFEM) shown in Figure 4

the IIFEM only monotonic space fields can be addressed. Thus the governing equations and boundary conditions are still equations (1)-(18) and the expansions of the unknowns are still those of equations (19) and (20), but the $x$-co-ordinates of equation (20) do not necessarily span isotherms. Figure 7 shows these arrangements and their differences from those of Figure 4 as the 
Newton iteration progresses. For example, the temperatures $T_{1,3}$ and $T_{2,3}$ are chosen to be between the known inlet temperature $T_{0,3}$ and the known solidification temperature $T_{\mathrm{s}}$ by linear interpolation. Upon convergence the corresponding nodes change position from their initial guess to where indeed the temperatures are $T_{1,3}$ and $T_{2,3}$ in order to satisfy the energy equation. With this modification, situations similar to that of Figure 5(b) at high Reynolds and/or Peclet numbers can be accommodated.

The governing equations, weighted integrally with the basis functions, result in the following continuity, $R_{\mathrm{C}}^{i}$, momentum, $R_{\mathrm{M}}^{i}$, and energy, $R_{\mathrm{E}}^{i}$, residuals:

$$
\begin{aligned}
R_{\mathrm{C}}^{i} & =\int_{V} \nabla \cdot \mathrm{u} \psi^{i} \mathrm{~d} V=0, \\
R_{\mathrm{M}}^{i} & =\int_{V}[\operatorname{Reu} \cdot \nabla \mathbf{u}+S t \mathbf{k}-\nabla \cdot(-p \mathbf{I}+\mathbf{T})] \phi^{i} \mathrm{~d} V=0, \\
R_{\mathrm{E}, \mathrm{L}}^{i} & =\int_{V}\left(P e_{\mathrm{L}} \mathbf{u} \cdot \nabla T-\nabla \cdot \nabla T-B r \Phi_{\mathrm{v}}\right) \phi^{i} \mathrm{~d} V=0, \\
R_{\mathrm{E}, \mathrm{S}}^{i} & =\int_{V}\left(P e_{\mathrm{S}} \frac{\partial T}{\partial x}-\nabla \cdot \nabla T\right) \phi^{i} \mathrm{~d} V=0,
\end{aligned}
$$

where $T=\nabla \mathbf{u}+(\nabla \mathbf{u})^{\mathrm{T}}$ and $\Phi_{\mathbf{v}}=\left[\nabla \mathbf{u}+(\nabla \mathbf{u})^{\mathrm{T}}\right]: \nabla \mathbf{u}$. By applying the divergence theorem in order to decrease the order of differentiation and to project the natural boundary conditions for the heat flux and the stress at the boundaries of the domain, equations (22)-(24) reduce to

$$
\begin{aligned}
R_{\mathrm{M}}^{i} & =\int_{V}\left[\operatorname{Re}(\mathbf{u} \cdot \nabla \mathbf{u}+S t \mathbf{k}) \phi^{i}+(-p \mathrm{I}+\mathrm{T}) \cdot \nabla \phi^{i}\right] \mathrm{d} V-\int_{S} \mathrm{n} \cdot(-p \mathrm{I}+\mathrm{T}) \phi^{i} \mathrm{~d} S=0, \\
R_{\mathrm{E}, \mathrm{L}}^{i} & =\int_{V}\left[\left(P e_{\mathrm{L}} \mathbf{u} \cdot \nabla T-B r \Phi_{\mathrm{v}}\right) \phi^{i}+\nabla T \cdot \nabla \phi^{i}\right] \mathrm{d} V-\int_{S} \mathrm{n} \cdot \nabla T \phi^{i} \mathrm{~d} S=0, \\
R_{\mathrm{E}, \mathrm{S}}^{i} & =\int_{V}\left(P e_{\mathrm{S}} \frac{\partial T}{\partial x} \phi^{i}+\nabla T \cdot \nabla \phi^{i}\right) \mathrm{d} V-\int_{S} \mathrm{n} \cdot \nabla T \phi^{i} \mathrm{~d} S=0 .
\end{aligned}
$$

Since essential boundary conditions will be applied to the inlet and to the wall for the $u$ - and $v$-velocities, equations (25) will be replaced by equations (5), (6), (8) and (9) accordingly. At the plane of symmetry the $v$-component of the momentum residual, equation (25), will be replaced by equation (16) and the surface integral of the $u$-component is zero according to equation (15). At the solidification front the momentum residual will be replaced by equations (12) and (13), which amounts to imposing the $u$ - and $v$-velocities of the liquid as essential boundary conditions. The energy residual of the liquid metal, equation (26), will be replaced by equation (7) at the inlet. The surface integrals of equations (26) and (27) will be replaced by equations (10) and (11) respectively. At the midplane the surface integrals of equations (26) and (27) vanish according to equation (17). At the solidification front the surface integrals of equations (26) and (27) will be replaced by equation (14) augmented by the term $n \cdot(\beta-1) \nabla T$ if the change in the heat capacities between molten and solid metal is not negligible.

The residuals are evaluated numerically by a nine-point Gaussian integration. A system of non-linear algebraic equations results, which is solved by Newton iteration according to the scheme

$$
\mathbf{q}^{(n+1)}=\mathbf{q}^{(n)}-\mathbf{J}^{-1} \mathbf{R}\left(\mathbf{q}^{(n)}\right)
$$


where $\mathbf{q}=\left[u_{1}, v_{1}, x_{1}, p_{1}, \ldots, u_{N}, v_{N}, x_{N}, p_{N}\right]$ is the vector of the unknowns and $\mathbf{J}=\partial \mathbf{R} / \partial \mathbf{q}$ is the Jacobian matrix of the residuals $\mathbf{R}$ with respect to the nodal unknowns $\mathbf{q}$. Notice that, owing to the inverse solution, the $x_{i}$ nodal positions and not the temperatures are the unknowns involved.

\section{RESULTS}

In order to examine the effect of the Reynolds and Nusselt numbers, a solution was first obtained at $R e=0$ and $N u=1$. Zero-order continuation was used to reach Reynolds numbers of the order of 1000 . The Newton iteration converged quadratically within four iterations, with a maximum nodal error less than $10^{-6}$, and the Reynolds number was increased by 50 each time a solution was obtained.

The inverse isotherm finite element method as developed by Alexandrou et al. ${ }^{1}$ was applied to solve the dimensionless Navier-Stokes equations augmented by the energy equation for the extrusion process of Newtonian fluids. Solutions were achieved for creeping flow $(R e=0)$ at Peclet numbers up to 0.2 and Nusselt numbers up to 0.5 . In Onoda's work, ${ }^{14}$ where the solidification front was calculated directly with the method of free surface parameterization, solutions were attained at Reynolds numbers up to 100, Peclet numbers up to 2 and Nusselt numbers up to 3 . In this work results have been obtained for Reynolds numbers up to 2000 , Peclet numbers up to 230 and Nusselt numbers up to 10. The upper limits of the Peclet and Nusselt numbers achieved are restricted by the values of the steel properties alone.

\section{The effect of the Reynolds number (casting rate)}

The Reynolds number was varied from 1180 to 1770 , which corresponds to casting rates from 1 to $1.5 \mathrm{~m} \mathrm{~min}^{-1}$, while all other process parameters were kept constant. As depicted in Figure 8, the meniscus attachment is relatively insensitive to the Reynolds number, while the shape and length of the solidification front are strong functions of the Reynolds number. This result agrees with the observation that small changes in the casting rate cause large changes in the length of the solidification front. ${ }^{18}$ Consequently, the cut-off point in the process must be changed appropriately, whenever the Reynolds number varies, so that a soft solid cast is cut. Moreover, as shown in Figure 9, the analysis of this work may provide empirical correlations which give the length of the solidification front as a function of the Reynolds number. These correlations predict that the length of the solidification front is proportional to the casting speed or the thickness of the solidified part proportional to the square root of residence time in the mould, in agreement with results obtained elsewhere. ${ }^{14}$ These simple correlations can be readily implemented in a control package algorithm which simplifies process control of metal casting.

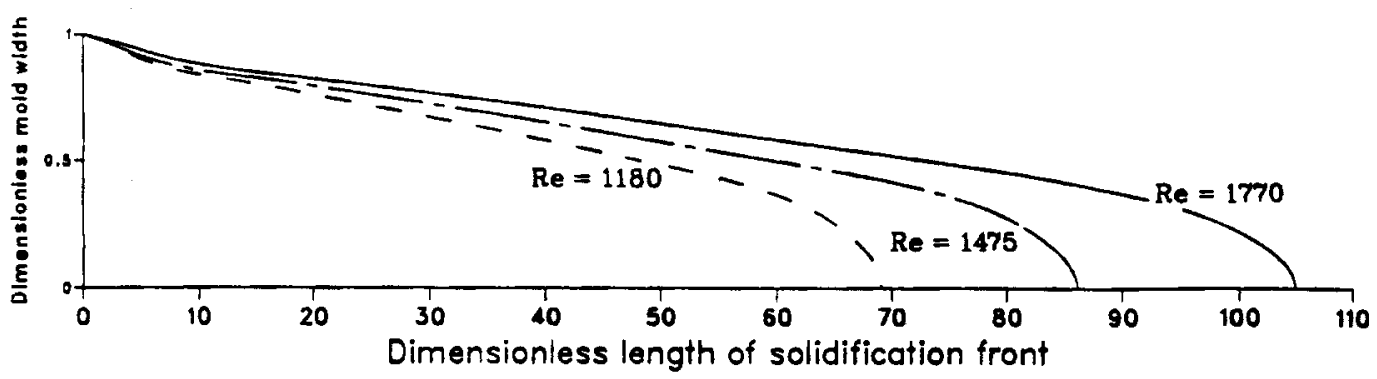

Figure 8. Change of solidification front with respect to Reynolds number at constant Nusselt numbers $\left(N u_{1}=2.95\right.$ along the solid wall and $N u_{2}=6.1$ along the free surface) and at constant Prandtl number $(P r=0.1135)$ 


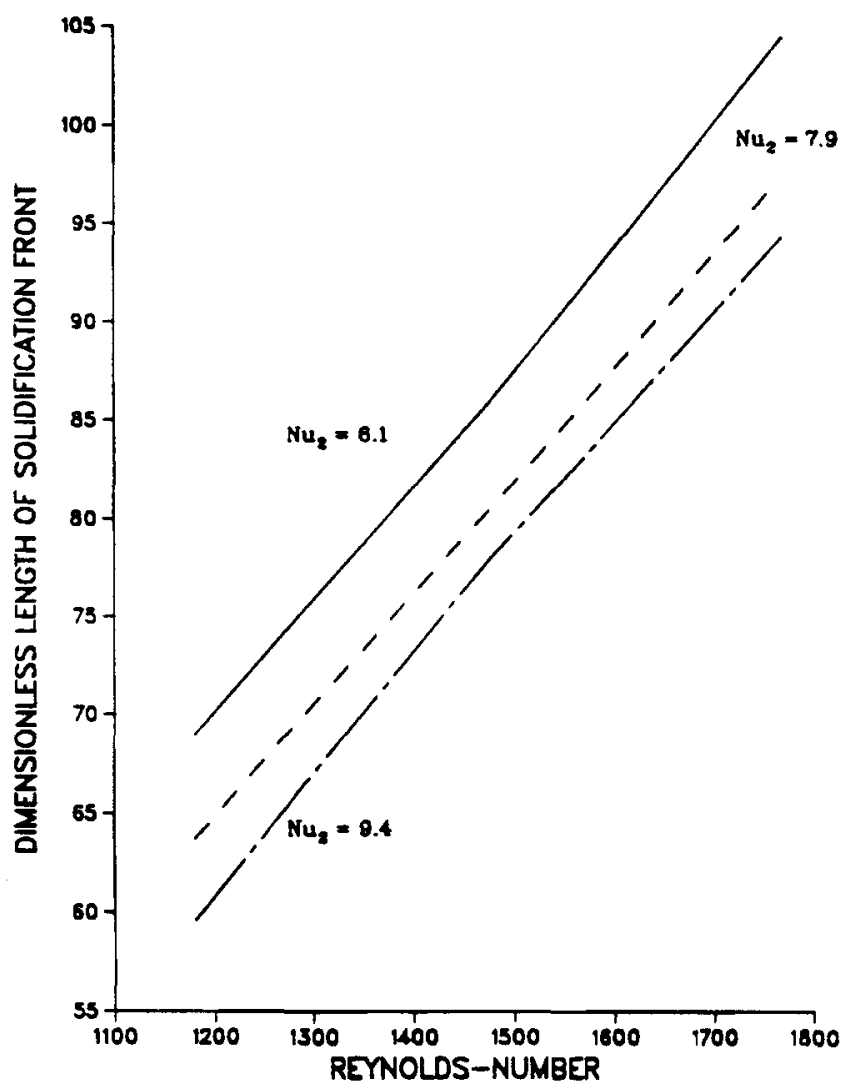

Figure 9. Empirical correlations between the dimensionless length of the solidification front, $L / W$, and the Reynolds number for three different Nusselt numbers $N u_{2}$ of the free surface and constant Nusselt number of the wall $\left(N u_{1}=2 \cdot 95\right)$, based on inverse finite element predictions

The effect of the Nusselt number (cooling rate)

The Nusselt number along the free surface was varied from 6 to 9 . All other parameters, i.e. the casting rate and the cooling rate along the wall, were kept constant. As depicted in Figure 10, the meniscus attachment is relatively insensitive to the Nusselt number, while the length of the solidification front is a weak function of the Nusselt number. Analogously to the previous results, the cut-off point of the process should also change according to a corresponding change in the cooling rate along the free surface. The exact dependence of the cut-off point on the cooling rate is shown in Figure 11. An empirical correlation can be established between the length of the solidification front and the cooling rate for process control purposes. Indeed, Figures 9 and 11 suggest a relation of the form

$$
\frac{L}{W}=0.06\left(\frac{\rho_{\mathrm{L}} V W}{\mu}\right)-2.33\left(\frac{h_{2} W}{k}\right),
$$

where $L$ is the length of the front, $W$ is the mould half-width and all other material properties and process parameters have been introduced in equations (1)-(18). 


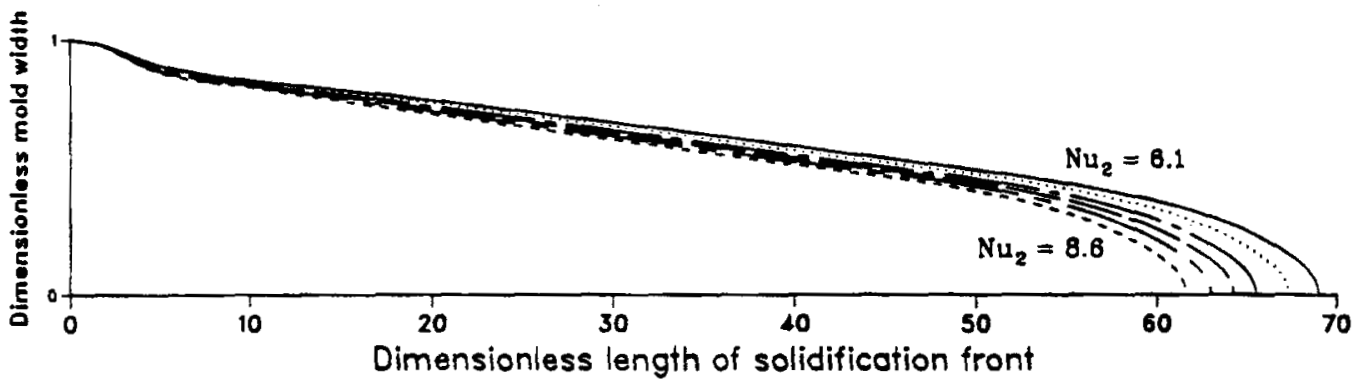

Figure 10. Change of solidification front with respect to Nusselt number of the free surface, $N u_{2}$, at constant Reynolds number $(R e=1180)$ and constant Nusselt number $\left(N u_{1}=2 \cdot 95\right)$ of the solid wall

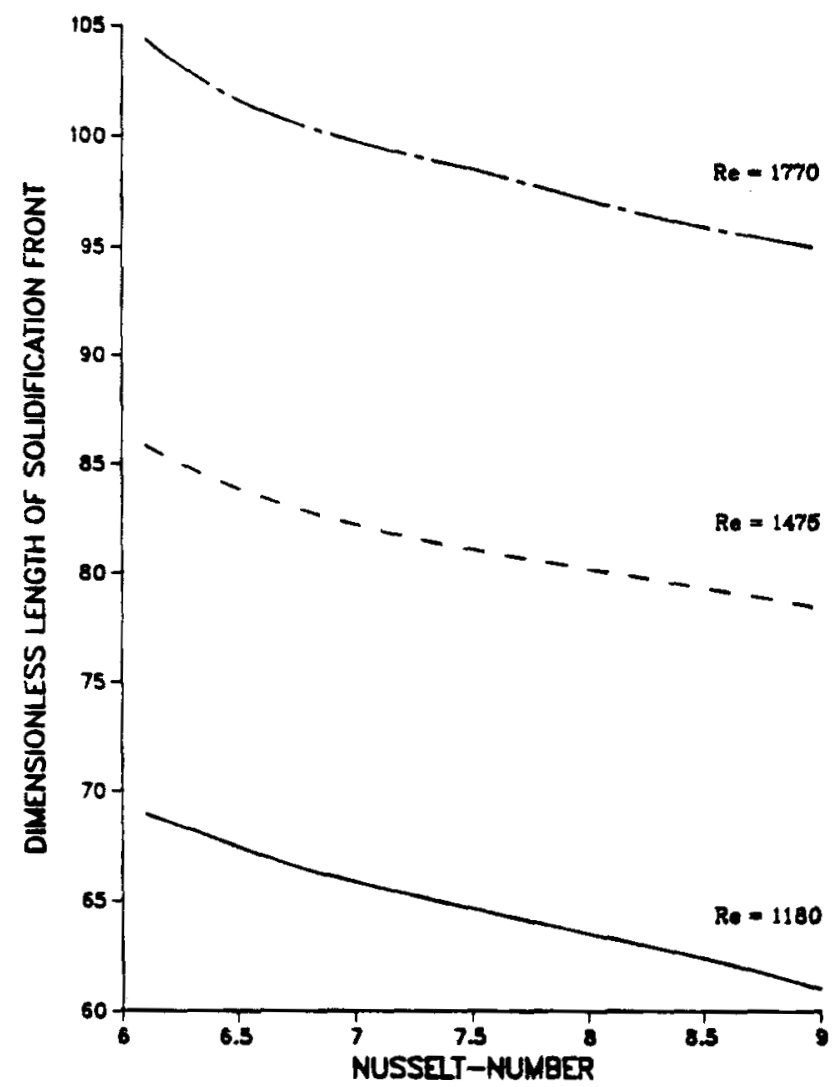

Figure 11. Empirical correlations between the dimensionless length of the solidification front, $L / W$, and the Nusselt number of the free surface for three different Reynolds numbers and constant Nusselt number of the wall $\left(N u_{1}=2.95\right)$

\section{The effect of temperature-dependent material properties}

In the discussion of the previous results the material properties were assumed to be independent of the temperature. A linear variation was assumed in $\rho_{\mathrm{L}}$, which is common in molten metals, ${ }^{24}$ given by

$$
\rho_{\mathrm{L}}(T)=\rho_{\mathrm{LO}}(\alpha-\beta T),
$$


where $\alpha=1.08$ and $\beta=0.08 .^{23}$ The length of the solidification front decreases when the density is temperature-dependent because cooling is enhanced by progressively lower Reynolds numbers; however, the dependence of the length of the solidification front on the casting rate is similar to that with temperature-independent density owing to the fact that the density of the molten metal changes slightly with temperature. Indeed, a comparison of the empirical correlations of the length of the solidification front with respect to the casting rate for temperature-independent and temperature-dependent molten steel density yields the same tendency, as shown in Figure 12.

The viscosity of the molten steel varies exponentially with temperature. ${ }^{24}$ In real casting operations the inlet temperature is at most $10 \%$ higher than the solidification temperature ${ }^{23}$ and the exponential dependence of the viscosity does not have any significant impact on the actual magnitude of the viscosity ${ }^{26}$ in as much as transition to solid occurs abruptly without an intervening solidification zone. However, if the inlet temperature is 1.5 times the solidification temperature, then the magnitude of the viscosity may vary up to $30 \%{ }^{25,26}$ and must be taken

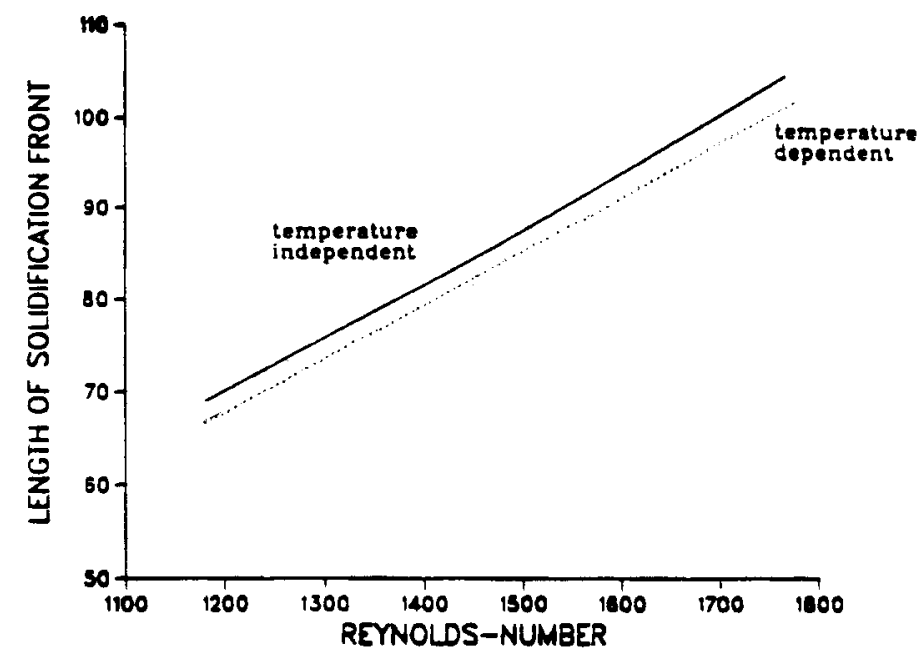

Figure 12. Comparison of the change of dimensionless length of the solidification front, $L / W$, when using the compressible $(\cdot)$ and the incompressible $(-)$ continuity equation for a casting rate of $1.25 \mathrm{~m} \mathrm{~min}^{-1}$ and constant Nusselt numbers along the free surface and the wall $\left(N u_{1}=2 \cdot 95, N u_{2}=6 \cdot 1\right)$

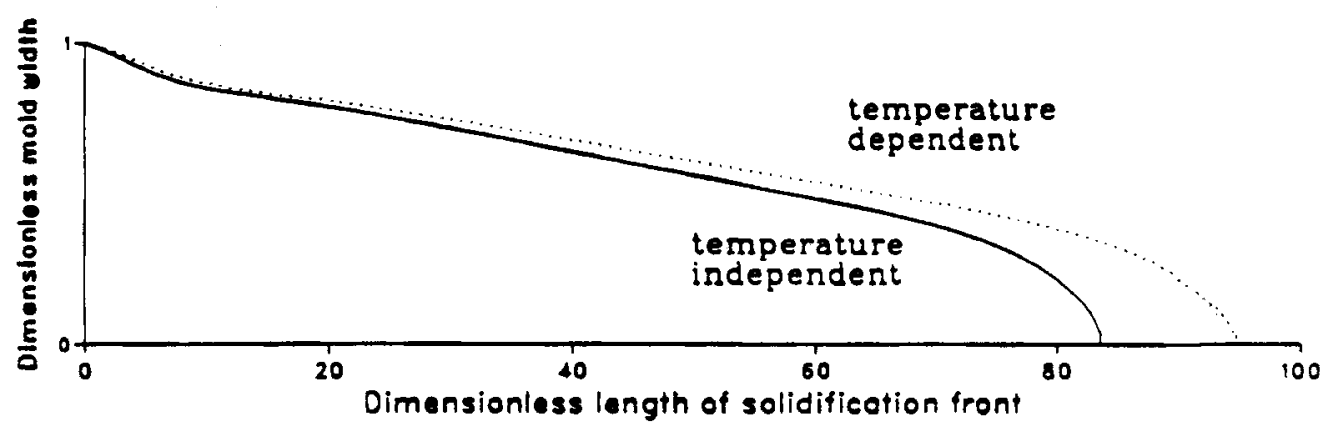

Figure 13. Velocity profiles at the meniscus attachment and downstream for Reynolds numbers in the range $100-1770$ and constant Nusselt numbers $\left(N u_{1}=2 \cdot 95,6 \cdot 1 \leqslant N u_{2} \leqslant 9 \cdot 4\right)$ 
into account. In Figure 13 it is shown how significantly the length of the solidification front changes when the exponential variation of the viscosity, given by

$$
\eta(T)=\eta_{0} \mathrm{e}^{\alpha / T},
$$

with $\alpha=1.07,{ }^{25}$ is not neglected. The length increases because at higher temperatures the viscosity decreases and the effective Reynolds number increases. The results are consistent with the computations with temperature-independent molten steel viscosity, where the solidification length increases with Reynolds number.

\section{Evaluation of one-dimensional analyses for process control}

Traditionally, process control is implemented under the assumption of plug flow downstream of the meniscus attachment, which leads to a simplified radial heat conduction equation to be solved. ${ }^{16-18}$ Calculations of the velocity profiles downstream of the meniscus attachment by this analysis are shown in Figure 14. The velocity profile is nearly parabolic just upstream of the meniscus attachment, being distorted in the vicinity of the wall, where, owing to the solid slip, a sudden acceleration of the adjacent fluid is caused. This profile prevails to over 5 to 30 diameters downstream, for Reynolds numbers in the range $100 \leqslant R e \leqslant 1770$ and Nusselt numbers in the range $6 \cdot 1 \leqslant N u_{2} \leqslant 9 \cdot 4$, where it becomes flat. This suggests that a two-dimensional analysis may provide a more accurate process control of continuous casting or, at least, adopted onedimensional analyses may need an a priori validation before implementation. Two-dimensional traditional analyses of metal casting compute the shape of the solidification front indirectly using Picard-like iterations which converge slowly and often not at all. The analysis here, with its inverse character and the use of Newton iteration with its fast and quadratic convergence, given a good initial estimate, is consistent with control purposes where the speed of simulation is of primary importance. Moreover, the Jacobian matrix contains vital information for linear stability analysis, first-order continuation and sensitivity analysis in general.

Unfortunately, there are not a lot of experimental data to compare the theoretical predictions of this work to. Some experiments have been performed for the continuous metal-casting process that suggest an approximate dependence of the thickness $d(x)$ of the solidified shell on the square root of the residence time $t$ in the mould: ${ }^{18}$

$$
d(x) \approx \sqrt{ } t
$$

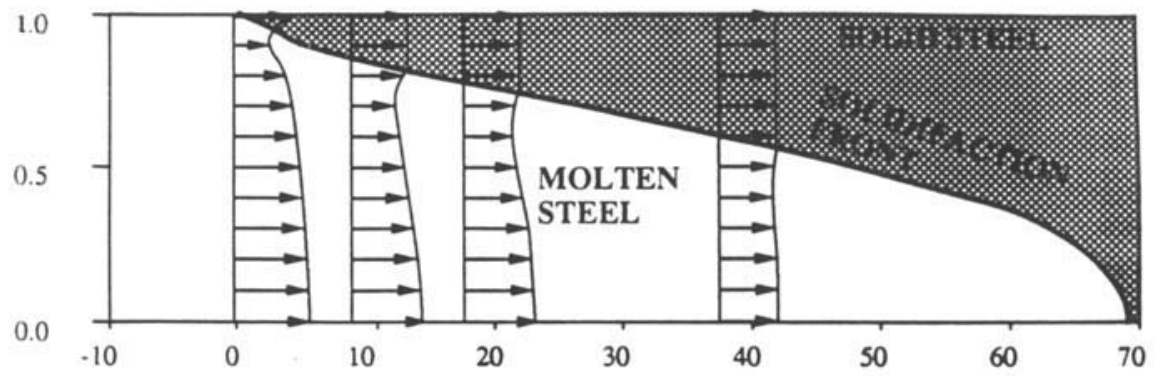

Figure 14. Comparison of the empirical correlations between the dimensionless length of the solidification front, $L / W$, and the Reynolds number for constant Nusselt numbers along the free surface and the wall $\left(N u_{1}=2 \cdot 95, N u_{2}=6 \cdot 1\right)$ between temperature-independent and temperature-dependent molten steel density at constant Prandtl number $\operatorname{Pr}=0 \cdot 1135$ 
where $t$ is the residence time, defined by

$$
t=x / V
$$

with $V$ the casting speed and $x$ the distance downstream from the attachment of the meniscus, where $d(x=0)=0$ and $d(x)$ is the thickness of the solidified shell at distance $x$ from the meniscus attachment.

According to equations (32) and (33),

$$
L=W^{2} V,
$$

i.e. experiments show that the length of the solidification front is proportional to the casting speed $V$ and the square of the mould thickness, in agreement with equation (29). Notice also that equations (32) and (33) are incorporated in the general equation (23).

There are sparse, incomplete experimental data and other theoretical results of one- and two-dimensional models that compute the actual shape and length of the solidification front in continuous casting of steel. ${ }^{26}$ These incomplete data are not conclusive because important parameters such as the cooling and casting rates are not given. Additionally, measurements of solidification fronts by autoradiography are still not able to penetrate fully the solidus isotherm, so that an experimental error of $20 \%$ is anticipated. Under these conditions a direct quantitative comparison with experimental results appears impossible. Yet, the experimentally observed dependence of the solidification length on the casting rate has been predicted accurately, as well as the sensitivity of the solidification front with respect to the Reynolds and Nusselt numbers. Also, Figure 14 provides a strong indication that approximate one-dimensional analyses may lead to incorrect results.

\section{CONCLUSIONS}

The recently developed inverse isotherm finite element method has been extended further in order to account for problems with large distortions of isotherms and elements. The method can be used effectively to solve a wide variety of problems where the position and shape of characteristic lines of the prescribed property are required. Additionally, the idea of inversion can be generalized to many processes where the common approach to a problem that relates cause to result is reversed; for example, instead of computing extrudate shapes from given die geometries, the problem may be reversed and the die design is sought to obtain target extrudate shapes. ${ }^{27}$ The process of metal casting has been analysed with this method under realistic process conditions. The shape and position of the solidification front in the continuous casting of steel as functions of the Nusselt and Reynolds numbers have been investigated. The analysis provides empirical correlations which give the length of the solidification front as a function of the casting speed and the cooling rate, validate experimental observations in metal casting and can be easily implemented with control package algorithms. Conventional one-dimensional approaches to continuous steel casting may need a priori validation by two-dimensional analyses along the lines of the present work.

\section{ACKNOWLEDGEMENT}

This research was supported by the Office of the Vice-President for Research of the University of Michigan. 


\section{APPENDIX: NOTATION}

$\begin{array}{ll}B r & \text { Brinkman number } \\ C_{1}, C_{2} & \text { Algebraic constants } \\ \mathbf{i} & \text { unit vector pointing in the positive } x \text {-direction } \\ \mathbf{I} & \text { identity matrix } \\ \mathbf{J} & \text { Jacobian matrix } \\ \mathbf{k} & \text { unit vector in the direction of gravity } \\ n & \text { algebraic exponent } \\ \mathbf{n} & \text { unit normal vector } \\ N u & \text { Nusselt number } \\ p & \text { pressure } \\ P e & \text { Peclet number } \\ \mathbf{q} & \text { vector of unknowns in Newton iteration } \\ \mathbf{R} & \text { residual of the Galerkin FEM } \\ \mathbf{R e} & \text { Reynolds number } \\ S t & \text { Stokes number } \\ S t e & \text { Stefan number } \\ \mathbf{t} & \text { unit tangential vector } \\ T & \text { temperature } \\ \mathbf{T} & \text { stress tensor } \\ u & \text { fluid velocity in } x \text {-direction } \\ \mathbf{u} & \text { velocity vector of fluid } \\ v & \text { fluid velocity in } y \text {-direction } \\ x, y & \text { Cartesian co-ordinate directions }\end{array}$

\section{Greek symbols}

$\begin{array}{ll}\alpha & \text { density ratio } \\ \beta & \text { heat conductivity ratio } \\ \phi & \text { biquadratic basis functions } \\ \Phi_{\mathrm{v}} & \text { viscous dissipation term } \\ \psi & \text { bilinear basis functions }\end{array}$

$\begin{array}{ll}\text { Superscripts } \\ i & \text { ith node in FEM } \\ \mathrm{T} & \text { transpose }\end{array}$

\section{Subscripts}

$\begin{array}{ll}\text { C } & \text { continuity equation } \\ \text { E } & \begin{array}{l}\text { energy equation } \\ \text { liquid }\end{array} \\ \text { L } & \text { momentum equation } \\ \text { M } & \text { solid } \\ \text { S } & \text { along the solid wall } \\ 1 & \text { along the free surface }\end{array}$




\author{
Mathematical symbols \\ $\partial$ \\ $\nabla$ \\ partial derivative \\ nabla operator
}

\title{
REFERENCES
}

1. A. N. Alexandrou, N. R. Anturkar and T. C. Papanastasiou, 'An inverse finite element method with an application to extrusion with solidification', Int. j. numer. methods fluids, 9, 541-555 (1989).

2. K. R. Reddy and R. I. Tanner, 'On the swelling of extrudate plane sheets', J. Rheol., 22, 661 (1978).

3. H. B. Phuoc and R. I. Tanner, 'Thermally induced extrudate swell', J. Fluid Mech., 98, 253 (1980).

4. E. Ben-Sabar and B. Caswell, 'Heat transfer effects in die-swell', J. Rheol., 25, 537 (1981).

5. B. P. Huynh, 'Some finite element studies of extrusion', J. Non-Newtonian Fluid Mech., 13, 1 (1983).

6. J. J. Derby and R. A. Brown, 'Finite element analysis of a thermal-capillary model for liquid encapsulated Czochralski growth', J. Electrochem. Soc. Solid State Sci. Technol., 132, 470 (1985).

7. C. Cuvelier and J. M. Driessen, 'Thermocapillary free boundaries in crystal growth', J. Fluid Mech., 169, 1 (1986).

8. W. E. Langlois, 'Convection in Czochralski growth melts', PCH: Phys.-Chem. Hydrodyn., 2, 245 (1981).

9. W. E. Langlois, 'A parameter sensitivity study for Czochralski bulk flow of silicon', J. Cryst. Growth, 56, 15 (1982).

10. J. R. Pearson, 'On the melting of solids near a hot moving interface with particular reference to beds of granular polymers', Int. J. Heat Mass Transfer, 19, 405 (1976).

11. J. M. Ortega and W. C. Rheinbolt, Iterative Solution of Nonlinear Equations in Several Variables, Academic Press, New York, 1970.

12. D. R. Lynch and W. G. Grey, 'Finite element simulation of flow in deforming regions', J. Comput. Phys., 36, 135 (1980).

13. H. M. Ettourney and R. A. Brown, 'Finite element methods for steady solidification problems', J. Comput. Phys., 49, 118 (1983).

14. M. Onoda (with L. E. Scriven), 'Continuous solidification of cast sheet with finite element method', M.Sc. Thesis, University of Minnesota, 1984.

15. O. Tsubakihara, 'Technologies that have made direct concentration of continuous casting and hot rolling possible', Transactions of Iron and Steel Institute of Japan, 60, 89 (1987).

16. W. H. Ray, Advanced Process Control, McGraw Hill, New York, 1981.

17. K. P. Michalek, J. E. Kelly and J. A. Dantzig, 'Modeling of in-mold heat transfer in continuous casting of steel', in S. Kou and R. Mehrabian (eds.), Modeling and Control of Casting and Welding Processes, p. 497, TMS-AIME, Warrendale, PA, 1986.

18. E. A. Mizikar, 'Mathematical heat transfer model for solidification of continuously cast steel slabs', Trans. TMSAIME, 239, 1747 (1967).

19. A. N. Alexandrou, 'An inverse finite element method for directly formulating free boundary problems', Int. j. numer. methods eng., 28, 2383 (1989).

20. J. Crank and R. D. Pahle, 'Melting ice by isotherm migration method', Bull. J. Inst. Math. Appl., 9, 12 (1973).

21. Y. Talmon, H. T. Davis and L. E. Scriven, 'Moving boundary problems in simple shapes by isotherm migration', AIChE J., 29, 795 (1983).

22. N. Malamataris, "Computer-aided analysis of flows on unbounded and moving domains: change phase fronts and liquid leveling', Ph.D Thesis, University of Michigan, 1990.

23. R. D. Pehlke, A. Jeyarajan and H. Wada, 'Summary of thermal properties for casting alloys and mold materials', University of Michigan, 12/82, NTIS, PB83-211003.

24. G. H. Geiger and D. R. Poirier, Transport Phenomena in Metallurgy, Addison-Wesley, Reading, MA, 1973.

25. T. Chapman, 'The viscosity of liquid metals', AIChE J., 12, 395 (1966).

26. J. K. Brimacombe, 'Design of continuous casting machines based on a heat-flow analysis: state-of-the-art review', $C a n$. Metall. Q., 15, 163 (1976).

27. K. R. J. Ellwood, 'Mechanics and stability of 3D jets', Ph.D Thesis, University of Michigan, 1991. 